\title{
Concepciones de lectura y escritura en un posgrado en Arquitectura: un acercamiento desde la perspectiva de la Alfabetización Académica
}

\author{
Conceptions of reading and writing in a graduate degree \\ program in Architecture: an approach from the perspective of \\ Academic Literacy
}

DOI: https://doi.org/10.32870/dse.v0i23.972

\section{Jésica Franco*}

\begin{abstract}
Resumen
Este artículo presenta los avances de resultados de una investigación que busca aportar a la discusión en torno de las prácticas de enseñanza y aprendizaje en posgrado, centrándose específicamente en la lectura y la escritura. Para esto, se ha asumido un enfoque sociocultural que coincide con la perspectiva Alfabetización Académica. Como objetivo general, el estudio plantea describir las concepciones de lectura y escritura de docentes, estudiantes y el programa de estudios de un posgrado en la disciplina de Arquitectura. Es un trabajo de corte cualitativo, descriptivo y transversal, que se lleva a cabo en una universidad pública del estado de Veracruz, México, y se divide en dos etapas metodológicas. Hasta el momento, se ha concluido la primera etapa, en la que se ha aplicado un cuestionario autodiligenciado de preguntas abiertas. Los resultados que aquí se presentan muestran que quienes tienen concepciones transmisivas entienden que leer y escribir son procesos de codificación y decodificación de mensajes escritos que sirven para obtener o transmitir información. Por el contrario, aquellos que cuentan con concepciones más cercanas a lo transaccional, conciben la lectura y la escritura como procesos dialógicos, creativos y de construcción de significados que sirven para reflexionar junto con otros y transformar el propio pensamiento.
\end{abstract}

Palabras clave: concepciones de lectura y escritura - escritura académica - alfabetización académica educación superior - formación para la investigación.

\section{Abstract}

This article presents preliminary results of a study that seeks to contribute to the discussion on teaching and learning practices in graduate school, focusing specifically on reading and writing. For this purpose, a sociocultural approach which coincides with the Academic Literacies perspective has been assumed. As a general objective, this research paper aims to describe the reading and writing conceptions of teachers,

* Maestra en Investigación Educativa. Especialista en lectura, escritura y educación. Líneas de investigación: Alfabetización académica, lectura crítica, escritura académicas y formación de investigadores. Colaboradora externa de la Maestría en Arquitectura, Universidad Veracruzana. México. jesicafranco@hotmail.com 
students, and the curriculum of a graduate program in the discipline of Architecture. It is a qualitative, descriptive and transversal study, conducted in a public university in the state of Veracruz, Mexico, and is divided into two methodological stages. The first stage, in which a self-administered questionnaire with open-ended questions was applied, has been completed. The results presented here show that those who have transmissive conceptions understand that reading and writing are processes of encoding and decoding written messages that serve to obtain or transmit information. On the contrary, those who have more transactional conceptions conceive reading and writing as dialogic, creative and meaning-building processes that serve to reflect with others and transform their own thinking.

Keywords: reading and writing conceptions - academic literacy - academic writing - higher education research training.

\section{Introducción}

Históricamente, la universidad ha establecido prácticas que la constituyen como una institución social particularmente poderosa. Algunas de esas prácticas son la lectura, la escritura y la investigación. Leer y escribir, en el ámbito académico, son formas de construir conocimiento (Zavala, 2011), pero, además, son formas de pensar, hacer y decir. James Paul Gee (2010) observa que las personas no solo leen y escriben textos, sino que hacen cosas con ellos y, generalmente, ese hacer con los textos involucra a otras personas.

Lave y Wenger (1991) apuntan que las prácticas sociales son siempre situadas y se componen de actividades periféricas y sustanciales, lo cual genera una tensión continua entre los diversos agentes que conforman una comunidad de práctica. Es decir, que el proceso de inclusión de los sujetos en una comunidad se da a través de la participación en las prácticas particulares de esa comunidad. Igualmente, Wenger (1998) señala que participar no necesariamente supone una relación colaborativa, sino que puede involucrar todo tipo de relaciones, tanto conflictivas como armoniosas, íntimas, políticas, competitivas o cooperativas. Además, la inserción en una comunidad a través de las prácticas genera una serie de creencias, valores y percepciones del mundo en el individuo que median su experiencia y, al mismo tiempo, transforman a la comunidad, por lo que el potencial transformador de la participación social va en ambos sentidos (Wenger, 1998). De esta manera, las personas conocemos y aprendemos participando en las diversas actividades sociales a partir de las cuales experimentamos y significamos el mundo. Por eso, si bien existen varios factores que influyen en nuestras formas de hacer con la lectura y la escritura, lo que efectivamente llevemos a cabo dependerá, también, de los significados construidos socialmente.

Desde una postura basada en la teoría crítica (Horkheimer, Adorno, 1994; Marcuse, 1993) que cuestiona el individualismo, la desigualdad y la dominación en favor del cambio social, pienso que la enseñanza de la lectura y la escritura, en cualquier instancia, debe tener por objetivo ayudar a reflexionar sobre qué hacemos o, mejor dicho, qué podemos hacer cuando lee- 
mos y escribimos. Por eso, creo que apropiarse críticamente de estas prácticas implica tanto conocer los elementos que intervienen en la producción del discurso, como saber utilizarlos deliberadamente para participar en las actividades valoradas, en este caso, por las comunidades académicas.

Hacia finales de 1990, en el contexto latinoamericano, se consideraba que los estudiantes que ingresaban a la universidad ya habían sido alfabetizados en los niveles educativos previos, por lo cual, contaban con las herramientas para leer y escribir como se lo demandara el superior (Carlino, 2005). Muy frecuentemente, se les exigían tareas donde demostraran que entendían los textos especializados de las disciplinas y que, además, podían escribir sobre o a partir de ellos para generar nuevas ideas; si bien esta idea no ha sido totalmente superada, en las últimas décadas, se ha logrado comprender la necesidad de ocuparse de la lectura y la escritura en la educación superior (Villaseñor López, 2013). Reconocer que la lectura y la escritura constituyen prácticas estratégicas que pueden favorecer o restringir el acceso de los estudiantes al aprendizaje dentro de las disciplinas destaca la importancia de la enseñanza de la lectura y escritura en la formación y enculturación académica (Padilla, 2019) de los estudiantes universitarios.

De acuerdo con lo anterior, a lo largo del documento utilizo el término alfabetizar para referirme al proceso que busca acompañar el aprendizaje de la lectura y la escritura de las personas y favorecer su autonomía e inserción en la sociedad (Pipkin, Reynoso, 2010). Partiendo de lo postulado por Paula Carlino (2013), entiendo que alfabetizar académicamente es acompañar y potenciar el proceso de apropiación y reflexión crítica de la lectura y la escritura en los estudiantes universitarios para favorecer su autonomía, participación y garantizar su acceso a las diversas comunidades académicas.

Así, el propósito de este artículo es presentar los avances de la primera etapa de una investigación que se enmarca en la teoría de la Alfabetización Académica (AA). A continuación, se enuncian la problemática y el objetivo general de la investigación, se desarrollan el enfoque teórico y un breve estado de la cuestión, se describe el proceder metodológico y se exponen los resultados obtenidos hasta el momento.

\section{Problemática de investigación}

A partir del proceso de democratización de los estudios superiores (Pérez, Natale, 2016), y con el objetivo de nivelar el desempeño académico de los estudiantes, han surgido los cursos de lectura y escritura en varias IES. En general, estos cursos se ubican en los primeros semestres, pues buscan facilitar el acceso de los estudiantes de nuevo ingreso a la cultura académica. Pero a pesar de los esfuerzos para favorecer la inclusión y permanencia, las instituciones también se enfrentan a la disyuntiva entre remediar la formación con la que muchos estudiantes llegan al nivel superior o enfocarse a enseñar las particularidades del discurso académico, lo cual implica dejar afuera a aquellos estudiantes que no consiguen el nivel deseado por la institución. Así, los 
esfuerzos por remediar las desigualdades con las que los estudiantes acceden al nivel superior aún no logran incidir en la permanencia o en los resultados.

Lo anterior puede identificarse al recurrir, por ejemplo, a los datos de la Comisión Económica para América Latina y el Caribe (CEPAL), que indican que, en promedio para esta región, el porcentaje de jóvenes entre 25 y 29 años que concluyó el nivel superior fue de $18 \%$, y en MéxiCo, apenas 7.6\% (CEPAL, 2018). Si bien no podemos decir que la única causa del abandono escolar es el rezago educativo en habilidades básicas como la lectura, parece ser que los programas compensatorios tampoco ayudan a resolver los problemas de desfase de manera estructural.

Según el informe del Instituto Nacional para la Evaluación de la Educación (INEE, 2011), que evalúa el desempeño en lectura de los estudiantes que egresan del nivel medio superior a través de las evaluaciones PISA (2009) ${ }^{1}$ y Excale $(2010),{ }^{2}$ solo $14 \%$ puede localizar y organizar diferentes fragmentos de información que no corresponden a tareas superficiales, es decir, que no resultan evidentes en un texto, y pueden usar conocimientos formales o informales para elaborar hipótesis o evaluar críticamente lo que leen. En cuanto al desempeño en expresión escrita, apenas 5\% de los jóvenes son capaces de escribir un texto argumentativo con una estructura completa (presentación del tema, contextualización del lector, enunciación de tesis, argumentación pertinente, utilización de textos fuente sin tergiversar el contenido, y conclusiones). Por tal razón, es posible afirmar que los alumnos, al ingresar a la educación superior carecen de herramientas suficientes para afrontar el reto que implica leer y escribir para analizar, resolver problemas, tomar decisiones o generar conocimientos.

La escritura adquiere mayor relevancia en los estudios de posgrado, ya que, hoy en día, en un mundo globalizado y una economía basada en el conocimiento, haber cursado estudios de posgrado es uno de los requerimientos más valorados a la hora de ingresar en el campo laboral. Varios investigadores han confirmado las dificultades que presenta a los estudiantes de posgrado la escritura del trabajo de tesis para poder titularse (Arnoux, 2009; Espino, 2015; Chois, Jaramillo, 2016; Peredo, 2016). Sin embargo, las indagaciones más recientes acerca de la concepción de la lectura y la escritura como prácticas sociales señalan que la problemática no se debe a las "deficiencias" que arrastran los estudiantes, sino a las concepciones sobre lectura y escritura que se basan en la idea de que leer y escribir son técnicas de codificación y decodificación de mensajes, procesos cognitivos individuales que pueden perfeccionarse a partir de la enseñanza de ciertas reglas gramaticales y fórmulas de contenido que actúan como guías que deben seguir los estudiantes. Como señala Carlino (2004), los docentes desconocen el valor epistémico de la lectura y la escritura:

1 Aplicación especial de las pruebas PISA en 2009 a una muestra de estudiantes del último grado de educación media superior; la muestra tuvo representatividad nacional y estuvo conformada por 6,724 estudiantes de 231 escuelas.

2 La muestra se diseñó para representar a estudiantes de último grado de la educación media superior en México. Se aseguró la representatividad de los tres modelos educativos (Bachillerato Profesional Técnico, Bachillerato Tecnológico y Bachilleratos Generales) y sus modalidades (CONALEP, CBTIS, COBach, privados y autónomos). En total participaron 13,175 alumnos. 
Tres representaciones difundidas justifican estas suposiciones. Por un lado, se concibe que la escritura es solo un canal para comunicar lo que se sabe y no una herramienta de análisis que requiere volver a pensarlo (Alvarado, Cortés, 2000; Castelló, 2000). Por otro lado, y como efecto de lo anterior, se cree que redactar es una labor instantánea: sabiendo lo que se quiere decir, solo hace falta hacerlo por escrito. Finalmente, como ha advertido Russell (1990), se presupone que escribir es una técnica básica, la cual, una vez adquirida, sirve para poner sobre el papel cualquier conocimiento disciplinar. De este modo, el potencial epistémico de la producción escrita, la noción de que escribir es reescribir y la existencia de especificidades en los textos empleados por cada dominio del saber resultan ignorados. Con aquellas premisas cuestionables, las dificultades de redacción de los universitarios se adjudican tautológicamente a sus incompetencias, y la responsabilidad de ello, a los niveles educativos previos.

A su vez, los docentes tampoco están acostumbrados a reflexionar con sus estudiantes sobre la utilización de dichas prácticas en sus respectivas disciplinas, lo cual correspondería al desarrollo de una alfabetización académica (Carlino, 2013). Si consideramos, como señalan Morales y Cassany (2008), que cada género discursivo y cada ámbito presentan nuevos retos a los usuarios de la cultura letrada, parece lógico y necesario que las universidades tengan la responsabilidad de ayudar a los estudiantes a comprender los textos propios de su disciplina para que logren transitar con éxito su recorrido académico y constituirse como miembros activos y participativos en sus respectivas comunidades.

En este marco, es fundamental un análisis que nos permita conocer, en primer lugar, cómo conceptualizan la lectura y la escritura los estudiantes y docentes, y, en segundo lugar, cuáles son y cómo construyen esas prácticas. De esta manera, podríamos tomar como punto de partida lo que las personas saben para seguir construyendo su saber.

En general, las investigaciones realizadas respecto de esta problemática se enfocan en las áreas disciplinares de Humanidades y Ciencias Sociales (Carlino, 2005; Hernández, 2012; Difabio de Anglat, 2013; Navarro, 2017; Seide, Natale, 2017). Sin embargo, las indagaciones que consideran las áreas técnicas, como el caso de Arquitectura, ${ }^{3}$ son aún incipientes y solo se centran en hacer evidente la dificultad que los estudiantes de estas disciplinas tienen para producir textos académicos. La arquitectura, entendida como comunidad disciplinar, cuenta con formas de pensar, hacer y decir específicas, pero son escasos los estudios que se han realizado respecto de las prácticas de lectura y escritura en esta disciplina. Así lo apuntan Castaño Perea y de la Fuente Prieto (2013: 302) al hablar de los lenguajes específicos que se originan en las disciplinas técnicas:

Frecuentemente estos lenguajes propios esconden una condición técnica necesaria para la mayor precisión de los conceptos, pero en otras ocasiones solo son excusa para establecer los cotos de quien

3 La carrera de Arquitectura en la universidad en la cual se lleva a cabo este estudio se ubicada dentro del área técnica.

Diólo os sobre Educación 
pertenece a un determinado clan o tribu, alejando de la comunicación a todo aquel que no hable el mismo idioma. Esta situación, ya estudiada en varias disciplinas, con numerosa literatura científica al respecto (Castelló, Donahue, 2012; Hafner, 2013), en cambio, ha sido sistemáticamente olvidada en el caso específico del lenguaje del arquitecto.

Debido a lo anterior, y a la ausencia de indagaciones cualitativas sobre las prácticas de lectura y escritura en esta disciplina, la investigación en la que se enmarca este artículo tiene por objetivo general: analizar y describir las concepciones sobre las cuales se construyen las prácticas de lectura y escritura en un posgrado en Arquitectura.

\section{Enfoque teórico-conceptual}

La definición de AA de la cual parto es la que formuló Paula Carlino (2013: 27):

Sugiero denominar "alfabetización académica" al proceso de enseñanza que puede (o no) ponerse en marcha para favorecer el acceso de los estudiantes a las diferentes culturas escritas de las disciplinas. Es el intento denodado por incluirlos en sus prácticas letradas, las acciones que han de realizar los profesores, con apoyo institucional, para que los universitarios aprendan a exponer, argumentar, resumir, buscar información, jerarquizarla, ponerla en relación, valorar razonamientos, debatir, etcétera [...]. Conlleva dos objetivos que, si bien relacionados, conviene distinguir: enseñar a participar en los géneros propios de un campo del saber y enseñar las prácticas de estudio adecuadas para aprender en él. En el primer caso, se trata de formar para escribir y leer como lo hacen los especialistas; en el segundo caso, de enseñar a leer y a escribir para apropiarse del conocimiento producido por ellos. [...] alfabetizar académicamente equivale a ayudar a participar en prácticas discursivas contextualizadas.

Esta definición, a diferencia de la presentada anteriormente por la autora (cfr. Carlino, 2003), incluye explícitamente el rol de los docentes e instituciones educativas para evitar "dar a entender que alfabetizarse académicamente es un asunto que concierne solo a los alumnos" (Carlino, 2013: 45).

Vale, asimismo, diferenciar alfabetización de literacidad y de cultura escrita. Para Pipkin y Reynoso (2010), alfabetizar es el proceso por el cual se contribuye a que los sujetos avancen en el dominio de la lectura y la escritura. Destacan que este proceso es inacabable, es decir, siempre se puede seguir aprendiendo a leer y escribir mejor, independientemente de la edad o nivel de escolaridad. Daniel Cassany (2006: 38) señala que la literacidad (o las prácticas letradas) es todo aquello que se relaciona con el uso del alfabeto: "desde la correspondencia entre sonido y letra hasta las capacidades de razonamiento asociadas a la escritura". La literacidad -traducción literal del inglés, literacy- incluye las formas de hacer, pensar y decir, las prácticas socioculturales, donde conocimiento y contexto se afectan recíprocamente. Emilia Ferreiro (2007: 27) denomina cultura escrita al resultado de "los procesos de reconstrucción por el cual el sistema 
de marcas social y culturalmente constituido se transforma en la propiedad colectiva de cada nueva generación".

Todas estas definiciones convergen en que leer y escribir son prácticas sociales y culturales, que varían según el contexto de actividades, que se transforman constantemente, que generan pertenencia e identidad, y que no están exentas de conflicto. Es decir, prácticas que hacen a diversas y variadas formas de entender el mundo. Por eso no es extraño encontrar literatura en la que estos términos se utilicen de manera intercambiable.

La AA sugiere que la inserción en una comunidad disciplinar y profesional está invariablemente condicionada por las habilidades comunicativas y epistémicas que los jóvenes universitarios logren desarrollar a lo largo de su formación, y de las oportunidades que las instituciones les brinden al acompañarlos en ese proceso de enculturación ${ }^{4}$ (cfr. Carlino, 2005; Díaz, 2006, Padilla, 2019) a través de planes y programas de estudio que incluyan cursos de escritura académica en la especialidad, y diversas formas de apoyo para el mejoramiento de sus competencias discursivas (Sánchez Camargo, 2016: 47).

Escribir para aprender, reflexionar, comunicar o acreditar significa que enseñar escritura en la universidad incluye: aprender contenidos disciplinares, reflexionar sobre los fines y estructuras de los discursos propios de cada espacio disciplinar, evaluar y acreditar los aprendizajes mediante instrumentos consensuados y transparentes, desnaturalizar los conceptos, prácticas y expectativas de los diferentes ámbitos, y expresar creativamente las perspectivas e hipótesis que pueden explicar y mejorar la sociedad en la que vivimos (Navarro, 2017). Sin embargo, como se ha destacado en varias investigaciones, existe un desconocimiento generalizado acerca de qué implica leer y escribir en cada disciplina. Esto se verifica en el discurso de los directivos, docentes, e incluso, estudiantes, a la hora de conceptualizar sus prácticas de lectura y escritura (Carlino, 2013; Navarro, 2012; López, 2013; Sánchez, 2016).

La importancia de comprender las concepciones que subyacen las prácticas de lectura y escritura en la educación superior se relaciona con su función mediadora en la producción de discursos académicos y científicos. Si lográsemos entender cómo se construyen estas concepciones -muchas veces implícitas, incluso, para los mismos actores-, y distinguir qué factores influyen en ese proceso de construcción, podríamos ayudar a los estudiantes a reflexionar sobre ellas y generar mejores estrategias para que puedan apropiarse críticamente de las prácticas de su comunidad.

En México, existen aportes escasos, pero valiosos, en investigación sobre concepciones en escritura académica (Hernández, 2008, 2012; Hernández, Rodríguez, 2018; Gaeta et al., 2020), y el desarrollo de líneas de investigación al respecto es prácticamente inexistente. Esto implica una desventaja en la comprensión de qué factores intervienen en la calidad de las producciones escritas académicas y en el diseño e implementación de estrategias para la enseñanza de la escritura en el contexto universitario (Zanotto, 2018).

4 Entiendo enculturación como las formas de entender, compartir, adoptar, e incorporar prácticas del entorno cultural.

Diólo@os

sobre Educación

año 12 | número 23 | julio-diciembre 2021 | ISSN 2007-2171 
Según Hernández Rojas (2012), las investigaciones sobre esta noción se interesan por dos planos diversos aunque complementarios: las que refieren al plano individual, que intentan demostrar la existencia de una relación causal entre creencias y modos de escribir, y las que refieren al plano colectivo, que intentan demostrar la existencia de una relación causal entre creencias y prácticas de escritura, que señalan que las creencias en torno al modo de escribir se forjan a partir de las interacciones de los sujetos en una comunidad determinada. Es decir, las creencias son construidas por tipos de prácticas que tienen las comunidades. Sin embargo, como bien señalan Hernández Rojas y Rodríguez Varela (2018), hace falta mayor investigación para comprender esta última relación.

En función de lo anterior, para esta investigación se abordó la teoría de modelos implícitos de los procesos de lectura y escritura, que se refieren a diferentes patrones de compromiso entre lectores y escritores. Para Schraw y Bruning (1996), los modelos implícitos de lectura refieren a un conjunto de creencias epistémicas que no necesariamente son conscientes y que tienen que ver con dónde se considera que está el significado de un texto. En sus primeras investigaciones, evaluaron las creencias lectoras de los estudiantes universitarios y les pidieron que leyeran un cuento y escribieran un comentario. Los estudiantes con creencias vinculadas al modelo transaccional incluyeron una mayor cantidad de evaluaciones críticas y reacciones personales en sus productos escritos. En un trabajo posterior, White y Bruning (2004) evaluaron las creencias de escritura y descubrieron que los estudiantes con creencias de escritura transaccionales producían composiciones escritas de mayor calidad, donde integraban contenido crítico e ideas personales. Estos autores advierten que los modelos son independientes entre sí, por lo que estar de acuerdo con los supuestos de uno de ellos no determina el acuerdo con los supuestos del otro.

Así, el modelo transmisional se basa en la creencia de que el significado de un texto es independiente del lector o escritor y que la información se transmite desde el autor o el texto a la memoria del lector. Quienes se acercan más al modelo transaccional creen que el significado del texto está en la mente del lector o escritor, y por eso debe construirse activamente por los lectores o escritores integrando su propio pensamiento en el proceso. Por lo tanto, el modelo transmisional predispone a lectores y escritores a ser tomadores pasivos de significado en lugar de constructores activos de significado, mientras que mantener creencias consistentes con el modelo transaccional conduce a un compromiso más crítico y personal durante el proceso de lectura y escritura; en otras palabras, los modelos de transacción pueden predisponer a lectores y escritores a una variedad de procesos constructivistas.

Las teorías implícitas de la escritura también pueden recibir influencia de la comunidad en la que se desarrollan las prácticas. En muchos casos ya estudiados, las tareas de escritura que se practican en la universidad tienden a ser tradicionales y no se empatan con la noción de herramienta para el desarrollo de aprendizajes, a pesar de que los profesores universitarios reconozcan su potencial para aprender en las disciplinas (Villalón, Mateos, 2009; Castelló et al., 
2011; Solé et al., 2005). Asimismo, los estudios reportan las serias dificultades de los alumnos para hacer un uso epistémico de la escritura.

\section{Estado de la cuestión}

Las investigaciones sobre AA, a nivel internacional, se han incrementado en la última década. Una búsqueda rápida en las bases de datos ${ }^{5}$ nos permite corroborar que, en 2010, las publicaciones de artículos, conferencias y libros sumaban alrededor de 100, en español, y 350 en inglés. Mientras que, en 2018, las publicaciones en español ascendieron a más de 500, y en inglés superaron las 940 .

Sin embargo, como ya he mencionado, la producción científica sobre cómo conciben las prácticas de lectura y escritura los distintos actores en el ámbito de la educación superior es aún incipiente. Dentro de estas, encontramos el trabajo sobre representaciones de escritura llevada a cabo por Monserrat Castelló y Mar Mateos (2015) con 1,044 estudiantes y 280 profesores de nueve universidades españolas, donde analizan los resultados observados en función de diferentes áreas disciplinares (Artes y Humanidades, Ciencias Sociales, Medicina, Ingeniería y Arquitectura). La metodología utilizada fue cuantitativa: se utilizó la European Writing Survey (EUWRIT), que cuenta con dos versiones, una para docentes y otra para estudiantes. En los resultados se observó que la representación sobre qué implica escribir bien, "los estudiantes valoraron como importantes, en mayor medida que los docentes, los aspectos relativos al contenido: objetividad, creatividad, argumentación, estructuración, pensamiento crítico, justificación en fuentes científicas" (Castelló, Mateos, 2015: 496).

En su trabajo de tesis doctoral, Ruth Villalón (2010) realiza tres estudios. El primero incluye estudiantes de secundaria, bachillerato y universitarios, de instituciones públicas en Madrid y Guadalajara (España), y diferencia dos tipos de concepciones: una epistémica y otra reproductiva, dentro de las cuales analiza varias facetas de escritura: usos y funciones, planificación y textualización, y revisión y modificaciones. Los objetivos del estudio 1 fueron: 1) identificar las concepciones sobre la escritura de los estudiantes de secundaria, bachillerato y universidad; 2) indagar cómo varían las concepciones en función de la faceta de la escritura explorada; y 3) conocer el efecto del nivel educativo, del género y del dominio de conocimiento sobre dichas concepciones. La muestra estaba compuesta por 202 alumnos de secundaria, 163 de bachillerato y 310 estudiantes universitarios. Se utilizó un muestreo accidental y se elaboró un instrumento con base en tres fuentes: las respuestas a un cuestionario previo sobre escritura de preguntas abiertas, algunos ítems de cuestionarios utilizados en otras investigaciones y el marco teórico sobre modelos de escritura (Cassany, 1999; Graham et al., 1993; Scardamalia, Bereiter, 1987), y teorías implícitas (White, Bruning, 2004; Pozo et al., 2006). Los resultados indican que mientras los alumnos de secundaria se encuentran más próximos a una concepción reproductiva, los

5 Búsqueda realizada el 21/07/ 2019 en la base de datos Dimensions, bajo las palabras clave "alfabetización académica" y "academic literacy". 
universitarios se acercan más a la concepción epistémica. No obstante, ni siquiera este grupo de estudiantes se muestra plenamente de acuerdo con la escala epistémica.

En Chile, Navarro et al. (2020) indagan en las representaciones sociales sobre escritura académica y su enseñanza en estudiantes de nuevo ingreso en seis áreas disciplinares: Artes, Humanidades, Ingenierías, Ciencias de la Salud, Pedagogías en Ciencias y Ciencias Sociales. El estudio, de corte cualitativo, analizó 360 respuestas abiertas de 180 informantes. Las preguntas apuntaron a las dificultades y desafíos y a la enseñanza de la escritura con el objetivo de generar evidencias que puedan orientar políticas institucionales y pedagógicas de acompañamiento en el aprendizaje y la enseñanza de la escritura. Los resultados muestran que para los estudiantes la escritura representa un mayor desafío en los aspectos de gestionar los puntos de vista, tanto de las distintas fuentes como de los posibles lectores y de sus propias perspectivas, y desarrollar sus propias ideas en un texto. Consideran difícil acomodar sus aportes críticos o personales a las expectativas de la disciplina, de los profesores y de la institución. Con respecto a su enseñanza y aprendizaje, los estudiantes consideran que la escritura se aprende a partir de la práctica, acompañados de la enseñanza y sugerencias de profesores y tutores.

En México, las investigaciones acerca de las teorías implícitas en estudiantes universitarios llevadas a cabo por Hernández Rojas (2012) y Hernández Rojas y Rodríguez Varela (2018) dan cuenta de las diferencias entre disciplinas: en el caso de los estudiantes de Química, existen concepciones implícitas predominantes de tipo preconstructivo, es decir, intentan generar una escritura transformadora que no se logra del todo, pero que tampoco se podría considerar que corresponde a una concepción de tipo receptiva-reproductiva. En cambio, en los estudiantes de Letras se encontraron concepciones implícitas de tipo constructivo, caracterizada por el autor como aquella que aprecia la complejidad de la tarea de escritura, que tienen en cuenta las distintas variables que se ponen en juego y que identifica sistemáticamente el contenido de lo retórico-comunicativo, con lo cual se muestra una mayor actividad reflexiva y autorreguladora en la tarea de escritura. Con base en lo anterior, Hernández (2012: 57) sostiene que:

La pertenencia a comunidades académicas-disciplinares que cuentan con un diferente discurso para hablar sobre los textos y sobre sus funciones. Mientras que los estudiantes de Letras pertenecen a una comunidad académica que con seguridad cuenta con un discurso más rico y útil para hablar sobre los textos, sobre los elementos básicos de la lectura y escritura y sobre sus funciones comunicativas, retóricas, estéticas, etc., los estudiantes de la carrera de Química no parecen tener a su disposición un discurso de este tipo.

Asimismo, Hernández (2012) aclara que sus resultados no coinciden con algunos estudios previos en los que se detectan concepciones más de tipo reproductivo en estudiantes universitarios, que concepciones constructivas (Villalón, Mateos, 2009), aunque sí comparten hallaz- 
gos en lo referente a que la mayoría de los estudiantes "no considera a la escritura como una herramienta potencial para el desarrollo de aprendizajes", es decir, no consideran su función epistémica.

El estudio exploratorio de Gaeta et al. (2020) tuvo por objetivo identificar el tipo de concepciones de escritura académica en estudiantes de medicina en sus dimensiones epistémica y reproductiva. Fue desarrollado desde un enfoque cuantitativo y participaron 359 estudiantes, de primero a décimo semestres, de la Facultad de Medicina de una universidad localizada en la ciudad de Puebla (México). Se aplicó el "Instrumento de concepciones sobre escritura académica", basado en White y Bruning (2004) y Villalón (2010), con 35 ítems de respuesta tipo Likert. Los hallazgos resaltan que, en consonancia con la literatura (Villalón, Mateos, 2009), los estudiantes no presentan una plena conciencia de creencias de tipo epistémico, lo que implica, según las investigadoras, que en los jóvenes, a pesar de tener una visión más elaborada que los estudiantes de niveles educativos previos, aún subyace la concepción de reproducir información mediante la escritura. Sin embargo, en consonancia con otras investigaciones (Hernández, 2012, 2017; Hernández, Rodríguez, 2018; Villalón, 2010), los universitarios no manifiestan un completo acuerdo con las concepciones epistémicas, lo que supone una dificultad para la elaboración de ideas y de aprendizaje a partir de la escritura académica. Las autoras señalan que para futuras investigaciones, sería pertinente obtener información sobre las prácticas de escritura que se desarrollan en el contexto de enseñanza y aprendizaje de la medicina, como así también aplicar métodos cualitativos para explorar la percepción que tienen los estudiantes sobre dichas prácticas en la disciplina que estudian.

Las investigaciones han ahondado sobre cómo docentes y estudiantes conciben la lectura y la escritura en el contexto académico, tanto en el nivel medio-superior como superior y, en general, clasifican estas concepciones en dos extremos opuestos: uno más cercano a lo reproductivo (donde, en particular, la escritura se piensa como una herramienta para reproducir información) y otro más cercano lo epistémico (como herramienta para aprender). Estos estudios utilizan instrumentos de medición cuantitativos y muestras que incluyen diversas áreas disciplinares, aunque no ahondan en las prácticas de los sujetos. Por tanto, la investigación que presento busca establecer una complementariedad desde un enfoque cualitativo y en una disciplina que aún no ha sido explorada.

\section{Proceder metodológico}

La investigación de la cual se desprende este artículo es descriptiva y transversal. El posgrado en Arquitectura con el cual he trabajado pertenece a una universidad pública, en el estado de Veracruz. Es un programa con orientación en investigación, que cuenta con tres Líneas de Generación y Aplicación del Conocimiento (LGAC): Habitabilidad Residencial, Arquitectura, Ciudad y Ciudadanía, Teoría Crítica e Historia de la Arquitectura. El plan de estudios es escolarizado, tiene 
un perfil interdisciplinario y una estructura curricular flexible, constituida por experiencias educativas (EE) obligatorias y optativas. Además, desde agosto de 2011, el programa se encuentra registrado en el PNPC, por lo cual se rige por los lineamientos del CONACyT y mecanismos internos para asegurar su calidad.

\section{Sujetos-colaboradores}

La comunidad se conforma de: a) un Núcleo Académico Básico (NAB), compuesto por nueve docentes de tiempo completo; $b$ ) los estudiantes que integran las dos generaciones activas para el periodo enero-julio de 2020: octava (cursando su cuarto semestre) y novena (cursando su segundo semestre), formadas por 9 y 10 estudiantes, respectivamente.

Asimismo, el programa cuenta con un total de 18 EE equivalentes a 45 horas de teoría y 18 de práctica. Si bien, en un principio, me había propuesto trabajar con la totalidad de docentes del NAB y estudiantes de las dos generaciones mencionadas, las circunstancias de contingencia por Covid-19 (ocurridas en las fechas propuestas para la aplicación del cuestionario: abril de 2020) me obligaron a implementar las herramientas metodológicas solamente con sujetos voluntarios, ya que no pude ponerme en contacto con la totalidad de docentes y estudiantes. De esta manera, los sujetos colaboradores fueron 20 en total: 12 estudiantes (4 de octava generación y 8 de novena generación) y 8 docentes.

Los docentes participantes ( $75 \%$ mujeres y $25 \%$ hombres) fueron, en su totalidad, de nacionalidad mexicana. $75 \%$ tiene entre 50 y 64 años de edad y $87 \%$ cuenta con grado de doctor/a en el área de Arquitectura o Urbanismo. En cuanto a los estudiantes (58\% hombres y $42 \%$ mujeres), solamente uno de ellos era de nacionalidad extranjera. Sus edades comprendían entre 30 y 38 años (50\%) y entre 24 y 29 (50\%). 17\% ya contaba con estudios de posgrado. En la tabla 4 se puede observar un resumen de los datos mencionados.

\section{Diseño}

El diseño propuesto para esta indagación cualitativa se basa en el formulado por Benedetto Minacore (2003), quien utiliza una perspectiva plurimetódica para delimitar su problema de investigación, pues considera que los aportes de las diferentes técnicas y métodos permiten la triangulación para contrastar y comprobar la exactitud de los datos recolectados. La triangulación de métodos y actores (estudiantes, docentes y programa de estudios) permite aumentar la validez y confianza de la información obtenida y un mayor conocimiento del objeto de estudio. Es así que he dividido el proceder metodológico en dos etapas, de las cuales describiré la primera, que es la que se ha concluido.

En la primera etapa apliqué un cuestionario autodiligenciado con preguntas abiertas. Monje Álvarez (2011: 135) define el cuestionario autodiligenciado como un formato que llenan por escrito los propios sujetos de la investigación; la ventaja es que "reduce los sesgos ocasionados 
por la presencia del entrevistador y tiene un formato simple que facilita el análisis y reduce los costos de aplicación". Luego, a partir de las preguntas del cuestionario, realicé el análisis de las EE que componen el programa de estudios.

\section{Desarrollo y aplicación de cuestionarios}

Las preguntas desarrolladas para el cuestionario fueron abiertas y de opinión, pues se buscaba identificar semejanzas y diferencias entre los distintos actores, describir sus concepciones transmisivas y transaccionales (partiendo de las ya definidas por la teoría) e indagar en las prácticas de lectura y escritura que se correspondían con cada tipo. Asimismo, generé cuestionarios específicos para docentes y estudiantes. Ambos cuestionarios fueron validados en el mes de febrero de 2020 mediante un grupo conformado por tres exalumnos del posgrado y tres docentes de la Facultad de Arquitectura que no forman parte del NAB.

El proceso de validación de los cuestionarios duró dos semanas. Envié los cuestionarios por correo electrónico y me reuní de manera presencial con cada uno de los exalumnos y docentes para escuchar sus devoluciones. A partir de estas devoluciones, reformulé algunas preguntas y reagrupé otras. También organicé las preguntas en dos apartados: lectura y escritura en general, y lectura y escritura en el ámbito académico, con la intención de ver si las respuestas diferían según el contexto de referencia.

La implementación de los cuestionarios se llevó a cabo de manera virtual, a través de la aplicación Forms, de Microsoft. En esta plataforma se registraron automáticamente las respuestas recogidas durante el mes de abril de 2020, en un periodo de tres semanas. Obtuve 20 cuestionarios en total: 12 completados por alumnos y 8 por docentes. Para atender las consideraciones éticas de la investigación, incluí un apartado de consentimiento informado que detallaba los objetivos de la investigación y establecía que, tanto la información proporcionada como la identidad de los participantes, sería anonimizada.

\section{Proceso de categorización}

La elaboración de categorías y subcategorías fue uno de los elementos fundamentales de la investigación. De acuerdo con Cisterna (2005), las categorías y subcategorías pueden ser apriorísticas, es decir, construidas desde la teoría antes de comenzar la recolección de datos, o emergentes, que surgen del trabajo de campo. Asimismo, el autor recomienda elaborar un listado de herramientas conceptuales y operacionales antes de ingresar a campo, en especial, a los investigadores que no cuentan con gran experiencia. De esta manera, se facilita su tarea de indagación. Por lo tanto, para la primera etapa de este trabajo, decidí elaborar un listado de categorías apriorísticas, con el objetivo de que el ingreso a campo y la búsqueda de datos fueran más organizados. 
Las categorías que utilicé para la creación de las preguntas del cuestionario fueron construidas con base en las definiciones de modelos implícitos de lectura (Schraw, Bruning, 1996) y creencias de escritura (White, Bruning, 2004). Así, establecí dos categorías principales y doce subcategorías. Dentro de la categoría lectura, incluí las subcategorías: definición de lectura, rol del lector y significado del texto según creencias transmisivas, y según creencias transaccionales. Lo mismo hice con la categoría escritura (véase tabla 1).

Tabla 1 Categorías apriorísticas. Elaboradas con base en las definiciones de modelos implícitos de lectura (Schraw, Bruning, 1996) y creencias de escritura (White, Bruning, 2004)

\begin{tabular}{|c|c|c|}
\hline 过 & SUBCATEGORÍAS & DEFINICIONES \\
\hline \multirow{6}{*}{ 突 } & $\begin{array}{l}\text { Definición de lectura según } \\
\text { CreEnCIAS TRANSMISIVAS }\end{array}$ & Leer es analizar de manera objetiva la estructura y contenido del texto. \\
\hline & $\begin{array}{l}\text { Definición de lectura según } \\
\text { CREENCIAS TRANSACCIONALES }\end{array}$ & $\begin{array}{l}\text { Leer es un proceso inherentemente subjetivo más que un acto de recepción de las ideas del autor } \\
\text { o de traducción del significado del texto de la manera más objetiva posible. }\end{array}$ \\
\hline & $\begin{array}{l}\text { Rol de lector según } \\
\text { CREENCIAS TRANSMISIVAS }\end{array}$ & Énfasis en el autor. El lector es un receptor pasivo que extrae el significado previsto por el autor. \\
\hline & $\begin{array}{l}\text { Rol del lector según } \\
\text { CREENCIAS TRANSACCIONALES }\end{array}$ & $\begin{array}{l}\text { Énfasis en el lector. Los lectores interpretan el texto teniendo en cuenta sus propios objetivos y } \\
\text { propósitos personales dentro de un contexto particular. }\end{array}$ \\
\hline & $\begin{array}{l}\text { Significado del texto según } \\
\text { CREENCIAS TRANSMISIVAS }\end{array}$ & El significado está en el texto y debe ser extraído, de manera objetiva, por el lector. \\
\hline & $\begin{array}{l}\text { Significado del texto según } \\
\text { CREENCIAS TRANSACCIONALES }\end{array}$ & $\begin{array}{l}\text { El significado del texto es construido por el lector con respecto a su conocimiento previo del domi- } \\
\text { nio del tema, sus experiencias de lectura anteriores y sus objetivos situacionales. Por lo cual, un } \\
\text { texto significa cosas diferentes para diferentes lectores, independientemente de lo que el autor } \\
\text { pretendía o de lo que contiene el texto. }\end{array}$ \\
\hline \multirow{6}{*}{ 旁 } & $\begin{array}{l}\text { Definición de escritura según } \\
\text { CREENCIAS TRANSMISIVAS }\end{array}$ & $\begin{array}{l}\text { Conciben la escritura como una forma de transferir información de fuentes autorizadas al lector de } \\
\text { manera que se limita la forma en que las ideas del escritor se reflejan en el texto. }\end{array}$ \\
\hline & $\begin{array}{l}\text { Definición de escritura según } \\
\text { CREENCIAS TRANSACCIONALES }\end{array}$ & Conciben la escritura como un proceso subjetivo que pone en juego las emociones del escritor. \\
\hline & $\begin{array}{l}\text { Rol del escritor según } \\
\text { CREENCIAS TRANSMISIVAS }\end{array}$ & Un buen escrito es aquel en el que se hace el menor número de cambios posible. \\
\hline & $\begin{array}{l}\text { Rol del escritor según } \\
\text { CREENCIAS TRANSACCIONALES }\end{array}$ & $\begin{array}{l}\text { Se considera importante desarrollar un estilo de escritura distintivo (voz autoral). Hay una cons- } \\
\text { tante preocupación por revisar y mejorar lo escrito. }\end{array}$ \\
\hline & $\begin{array}{l}\text { Significado del texto según } \\
\text { CrEEnCIAS TRANSMISIVAS }\end{array}$ & Lo importante es que la información llegue al lector de manera objetiva. \\
\hline & $\begin{array}{l}\text { Significado del texto según } \\
\text { CREENCIAS TRANSACCIONALES }\end{array}$ & Se considera que escribir ayuda a comprender mejor lo que se está pensando. \\
\hline
\end{tabular}

\section{Análisis de cuestionarios}

Para el análisis de los datos surgidos de los cuestionarios, apliqué el Análisis Estructural del Discurso (AED), propuesto por Sergio Martinic (2006: 300) e inspirado en la semántica estructural de Greimas. Este tipo de análisis propone construir categorías a partir de ciertas reglas y procedimientos que buscan ordenar y clasificar el material discursivo con el fin de establecer los principios que organizan las concepciones de los sujetos sobre problemas y prácticas específicas: 
A través de las categorías se transforman los datos y el texto se reduce a unidades que puedan ser relacionadas, comparadas y agregadas a unidades mayores. Esta transformación implica, a su vez, pasar del texto y sentido literal a categorías y relaciones subyacentes entre categorías que producen un sentido y prácticas en contextos específicos.

A partir del proceso de codificación de los cuestionarios, para el cual utilicé el software Atlas.ti (versión 8.4.4), identifiqué ocho unidades básicas de sentido:

- Definición de lectura

- Definición de escritura

- Utilidad de la lectura en el contexto académico

- Utilidad de la escritura en el contexto académico

- Competencias necesarias para leer textos académicos

- Competencias necesarias para escribir textos académicos

- Prácticas de lectura académica

- Prácticas de escritura académica

Cada una de estas unidades, a su vez, se corresponde con los dos tipos de creencias establecidas desde la teoría: transmisivas y transaccionales. Por lo cual, durante el proceso de análisis trabajé a partir de la generación de relaciones de oposición y equivalencia (como indica el AED), de lo que resultaron 117 códigos, clasificados bajo cada una de estas unidades. Estos códigos emergentes me ayudaron a redefinir lo que se había planteado desde la teoría, es decir, las categorías apriorísticas con las que me había acercado a campo. Es por esto que considero el conjunto de estas ocho unidades como resultados preliminares (véase más adelante).

En el proceso de trabajo en Atlas.ti utilicé tanto la codificación por lista, como con la codificación in vivo, pues las respuestas, por sí mismas, sugerían palabras o frases que se correspondían con las unidades de sentido que había generado.

\section{Análisis del programa de estudios}

Las EE que conforman el programa de posgrado son 18. Cada experiencia consta de diversos apartados donde se describen sus contenidos. Por ejemplo, quiénes son los académicos que la imparten, cuál es la unidad de competencia que se busca desarrollar en los estudiantes, así como los saberes teóricos, heurísticos y axiológicos que debe alcanzar el alumno. Analicé las $\mathrm{EE}$ siguiendo los mismos pasos que utilicé para el análisis de cuestionarios. En general, el programa no mostró información que pudiera relacionarse con los tipos de creencias.

A continuación, presento los resultados obtenidos en esta primera etapa de investigación, concluida en el mes de junio de 2020. 


\section{Concepciones de lectura y escritura en posgrado}

El análisis de los datos me permitió describir, de manera preliminar, las concepciones transmisivas y transaccionales que tienen docentes, estudiantes y el programa, y establecer similitudes y deferencias. Como se puede apreciar en las figuras 1, 2 y 3, las concepciones que prevalecen en la comunidad académica estudiada son las transmisivas, tanto en docentes (56\%) como estudiantes (63\%).

Asimismo, estos resultados preliminares muestran que aquellos con concepciones transmisivas entienden que leer y escribir son procesos de codificación y decodificación de mensajes escritos, que sirven para obtener o transmitir información. Por el contrario, quienes cuentan con concepciones más cercanas a lo transaccional, conciben la lectura y la escritura como procesos dialógicos, creativos y de construcción de significados que sirven para reflexionar junto con otros y transformar el propio pensamiento. Estos hallazgos se detallan a continuación y se resumen en las tablas 2 y 3 .

Figura 1: Porcentajes de concepciones transmisivas y transaccionales en docentes

\begin{tabular}{|c|c|c|}
\hline \multirow[b]{3}{*}{ DOCENTE 1} & \multicolumn{2}{|c|}{ DOCENTES } \\
\hline & - Concepciones transaccionales & - Concepciones transmisivas \\
\hline & $70 \%$ & $30 \%$ \\
\hline DOCENTE 8 & $70 \%$ & $30 \%$ \\
\hline DOCENTE 6 & $69 \%$ & $31 \%$ \\
\hline DOCENTE 5 & $61 \%$ & $39 \%$ \\
\hline DOCENTE 7 & $33 \%$ & $67 \%$ \\
\hline DOCENTE 3 & $31 \%$ & $69 \%$ \\
\hline DOCENTE 4 & $11 \%$ & $39 \%$ \\
\hline DOCENTE 2 & $9 \%$ & $1 \%$ \\
\hline
\end{tabular}

Elaboración propia. 
Figura 2: Porcentajes de concepciones transmisivas y transaccionales en estudiantes

\begin{tabular}{|c|c|c|}
\hline \multicolumn{3}{|c|}{ ESTUDIANTES } \\
\hline Ec & cepciones transaccionales & Concepciones tran smisivas \\
\hline ESTUDIANTE 5 & 9 & $10 \%$ \\
\hline ESTUDIANTE 7 & $73 \%$ & $27 \%$ \\
\hline ESTUDIANTE 9 & $65 \%$ & $35 \%$ \\
\hline ESTUDIANTE 4 & $42 \%$ & $58 \%$ \\
\hline ESTUDIANTE 11 & $33 \%$ & $67 \%$ \\
\hline ESTUDIANTE 12 & $30 \%$ & $70 \%$ \\
\hline ESTUDIANTE 8 & $27 \%$ & $73 \%$ \\
\hline ESTUDIANTE 2 & $25 \%$ & $75 \%$ \\
\hline ESTUDIANTE 1 & $25 \%$ & $75 \%$ \\
\hline ESTUDIANTE 3 & $18 \%$ & $82 \%$ \\
\hline ESTUDIANTE 6 & $9 \%$ & $91 \%$ \\
\hline ESTUDIANTE 10 & $8 \%$ & $92 \%$ \\
\hline
\end{tabular}

Elaboración propia.

Figura 3: Porcentajes de concepciones transmisivas y transaccionales en docentes y estudiantes

\section{CONCEPCIONES EN DOCENTES Y ESTUDIANTES}

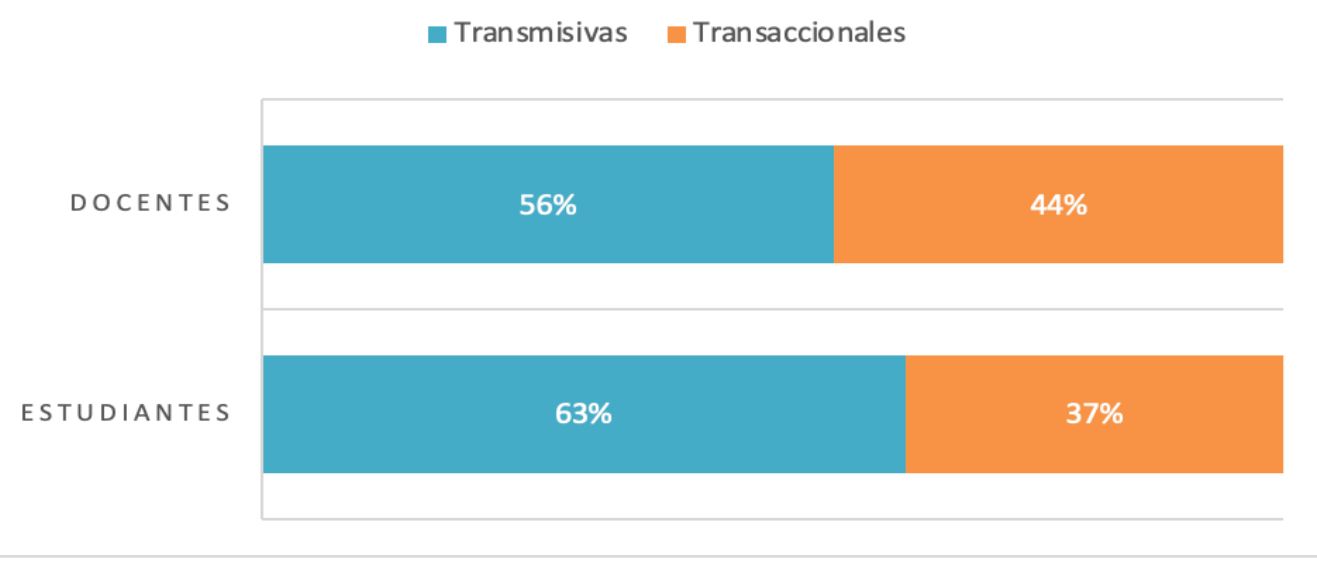

Elaboración propia.

Diólo oos

sobre Educación

año 12 | número 23 | julio-diciembre 2021 | ISSN 2007-2171 


\section{Concepciones transmisivas de lectura y escritura académicas}

Según las concepciones transmisivas de los docentes y estudiantes del posgrado en Arquitectura, la lectura se define como un proceso que requiere interpretar signos para obtener información. Así, en el contexto académico, leer sirve para adquirir conocimientos y, por lo tanto, para comprender el significado de un texto, para acceder a la tradición y conocimiento de su campo, el lector debe decodificarlo. Es decir, la lectura se entiende como un proceso individual, donde el lector es aquel que recibe, interpreta o interioriza lo que dice el texto.

Entre las competencias o conocimientos que se consideran necesarios para afrontar la lectura en este contexto, destacan: la comprensión lectora, la capacidad para jerarquizar información y los conocimientos del tema y lenguaje disciplinar. Algunas de las prácticas de lectura de estos docentes y estudiantes incluyen: identificar (subrayar) ideas principales, revisar la bibliografía para ampliar conocimientos y elaborar un resumen del texto.

Escribir, según las concepciones transmisivas, es transmitir lo que pensamos mediante palabras, por eso, lo que hacemos al escribir es codificar nuestros conocimientos para plasmarlos en un texto. En el contexto académico, escribir sirve para comunicar los resultados de una investigación o nuestras reflexiones respecto de un tema. Es decir, la escritura sirve para difundir conocimiento y se entiende que quien recibe ese conocimiento es un par.

En cuanto a las capacidades para escribir en contextos académicos, se consideran necesarias: la capacidad de análisis e investigación, el saber organizar la información (conocimiento del tema, de la estructura del texto y de la normativa de la lengua), la experiencia previa y el ser objetivo. Algunas de las prácticas de escritura relacionadas con estas concepciones son: organizar las ideas/información que se va a transmitir, redactar de manera clara y concisa, revisar la coherencia del texto. En la tabla 2, resumo los puntos anteriores. 
Tabla 2: Definición, utilidad, competencias necesarias y prácticas de lectura y escritura académicas según concepciones transmisivas

\begin{tabular}{|c|c|c|}
\hline \multicolumn{3}{|c|}{ CONCEPCIONES TRANSMISIVAS DE LECTURA Y ESCRITURA ACADÉMICAS } \\
\hline & Lectura & Escritura \\
\hline Definición & $\begin{array}{l}\text { Leer es un proceso individual que } \\
\text { consiste en interpretar signos (pala- } \\
\text { bras). Se considera que el lector debe } \\
\text { decodificar el texto para comprender } \\
\text { su significado, es decir, el lector reci- } \\
\text { be un mensaje escrito y lo asimila o } \\
\text { comprende para sí mismo. }\end{array}$ & $\begin{array}{l}\text { Escribir es transmitir ideas o pensa- } \\
\text { mientos mediante signos. Se considera } \\
\text { que el escritor debe codificar sus cono- } \\
\text { cimientos, plasmándolos en un texto. }\end{array}$ \\
\hline Utilidad & $\begin{array}{l}\text { La lectura, en el contexto académico, } \\
\text { sirve para adquirir información o co- } \\
\text { nocimientos y mejorar en el aspecto } \\
\text { profesional. }\end{array}$ & $\begin{array}{l}\text { La escritura, en el contexto académico, } \\
\text { sirve para comunicar los resultados de } \\
\text { una investigación o nuestras reflexio- } \\
\text { nes respecto de un tema. Es decir, la } \\
\text { escritura sirve para aportar informa- } \\
\text { ción y difundir conocimiento. }\end{array}$ \\
\hline $\begin{array}{l}\text { Competencias } \\
\text { necesarias }\end{array}$ & $\begin{array}{l}\text { Las competencias que, mayormente, } \\
\text { se consideran necesarias para leer } \\
\text { textos académicos son: la compren- } \\
\text { sión lectora, la capacidad para jerar- } \\
\text { quizar información y el conocimiento } \\
\text { del lenguaje disciplinar. }\end{array}$ & $\begin{array}{l}\text { Entre las competencias que se consi- } \\
\text { deran necesarias para escribir textos } \\
\text { académicos se incluyen: la capacidad } \\
\text { de análisis e investigación, el saber } \\
\text { organizar la información (conocimien- } \\
\text { to del tema, de la estructura del texto } \\
\text { y de la normativa de la lengua) y el ser } \\
\text { objetivo. Destacan, también, el tener } \\
\text { experiencia como escritor. }\end{array}$ \\
\hline Prácticas & $\begin{array}{l}\text { Entre las prácticas de lectura acadé- } \\
\text { mica, destacan: } \\
\text { - Leer siguiendo la estructura del } \\
\text { texto } \\
\text { - Revisar conceptos clave } \\
\text { Identificar (subrayar) ideas principa- } \\
\text { les } \\
\text { - Utilizar recursos para comprender el } \\
\text { vocabulario del texto } \\
\text { - Hacer varias lecturas } \\
\text { - Revisar la bibliografía para ampliar } \\
\text { conocimientos } \\
\text { - Elaborar un resumen del texto }\end{array}$ & $\begin{array}{l}\text { Las prácticas de escritura académica } \\
\text { incluyen: } \\
\text { - Organizar las ideas/información que } \\
\text { se va a transmitir. } \\
\text { - Redactar de manera clara y concisa, } \\
\text { con base en los autores leídos. } \\
\text { - Escribir un borrador. } \\
\text { - Escribir un texto final. } \\
\text { - Revisar coherencia del texto. }\end{array}$ \\
\hline
\end{tabular}

Elaboración propia.

\section{Concepciones transaccionales de lectura y escritura académicas}

Quienes cuentan con concepciones transaccionales de lectura entienden que leer es un proceso dialógico, creativo y de construcción de significados. Es decir, el lector no solamente recibe 
información, sino que participa en la construcción del significado de aquello que lee. Por lo tanto, para comprender un mensaje escrito, se debe conocer/indagar el contexto, pues leer se considera una práctica situada.

Según este tipo de concepciones, leer en el contexto académico sirve para establecer el diálogo, desarrollar un posicionamiento y fundamentar las ideas. Sirve también para pensar (analizar, cuestionar, problematizar, formar un criterio propio) y desarrollar ideas, incluso a partir de la escritura, por lo que la lectura también nos permite aprender a escribir. Dentro de los conocimientos necesarios para afrontar la lectura en este contexto, se señalan: la capacidad de reflexión sobre los propios intereses y motivaciones y la capacidad de análisis crítico (formular objetivos de lectura, reconocer la estructura e investigar el contexto del texto). Algunas prácticas de lectura relacionadas con las concepciones transaccionales son: establecer un objetivo de lectura, investigar contexto y autor y revisar las posturas teóricas que se discuten en el texto.

Respecto de la escritura, según las concepciones transaccionales, esta se define, ante todo, como una herramienta para comunicarse, por eso, al escribir debemos tener en cuenta quién será nuestro lector. Asimismo, quienes tienen estas concepciones indican que escribir es un proceso recursivo y creativo, a través del cual se puede pensar y construir conocimiento. Escribir nos permite transformar el propio pensamiento, motivar el diálogo, desarrollar un posicionamiento y generar conocimiento. Escribir también puede ser una herramienta de enseñanzaaprendizaje: un/a estudiante, al hablar del proceso de escritura de su tesis, expresa. "Incluso, a través del proceso de escritura, podemos ordenar las ideas, solucionar problemas y tomar decisiones".

Algunos de los conocimientos necesarios para escribir en el contexto académico son: la capacidad investigativa que tiene que ver con la competencia de lectura crítica (saber buscar fuentes relevantes, saber reconocer el propósito del escrito), la capacidad argumentativa, el conocimiento previo del tema que se aborda y la capacidad de construir una voz propia. Es importante resaltar la función creativa que los miembros de la comunidad disciplinar les adjudican a estas prácticas.

También es significativo el capital cultural, la humildad académica, el ser ético, y saber redactar de manera sencilla. Dentro de las prácticas de escritura relacionadas con las concepciones transaccionales, se mencionan: definir quién será el lector, generar mapas para desglosar ideas, plantear objetivos y definir un posicionamiento. En la tabla 3 resumo los puntos descritos anteriormente. 
Tabla 3: Definición, utilidad, competencias necesarias y prácticas de lectura y escritura académicas según concepciones transaccionale

\begin{tabular}{|c|c|c|}
\hline \multicolumn{3}{|c|}{ CONCEPCIONES TRANSACCIONALES DE LECTURA Y ESCRITURA ACADÉMICAS } \\
\hline & Lectura & Escritura \\
\hline Definición & $\begin{array}{l}\text { Leer es comprender, de manera situada, } \\
\text { un mensaje escrito. Asimismo, leer su- } \\
\text { pone un proceso dialógico y creativo de } \\
\text { construcción de significados por parte } \\
\text { del lector. }\end{array}$ & $\begin{array}{l}\text { Escribir es, ante todo, una herramienta para } \\
\text { comunicarse. Es decir, no se considera un } \\
\text { proceso individual, sino uno en el que el lector } \\
\text { está presente. Asimismo, escribir es un proce- } \\
\text { so recursivo y creativo, a través del cual pode- } \\
\text { mos pensar y construir conocimiento. }\end{array}$ \\
\hline Utilidad & $\begin{array}{l}\text { Leer, en el contexto académico, sirve } \\
\text { para establecer el diálogo, desarrollar } \\
\text { un posicionamiento y fundamentar las } \\
\text { ideas. Sirve también para pensar (ana- } \\
\text { lizar, cuestionar, problematizar, formar } \\
\text { un criterio propio) y desarrollar ideas. La } \\
\text { lectura, además, nos permite aprender } \\
\text { a escribir. }\end{array}$ & $\begin{array}{l}\text { Escribir, en el contexto académico, nos permi- } \\
\text { te transformar el propio pensamiento, motivar } \\
\text { el diálogo, desarrollar un posicionamiento y } \\
\text { generar conocimiento. A través del proceso } \\
\text { de escritura podemos ordenar las ideas, solu- } \\
\text { cionar problemas y tomar decisiones. Escribir } \\
\text { también puede ser una herramienta de ense- } \\
\text { ñanza-aprendizaje. }\end{array}$ \\
\hline $\begin{array}{l}\text { Competencias } \\
\text { necesarias }\end{array}$ & $\begin{array}{l}\text { Dentro de las competencias que se con- } \\
\text { sideran necesarias para leer textos aca- } \\
\text { démicos están: la capacidad de reflexión } \\
\text { sobre los propios intereses y motivacio- } \\
\text { nes, y la capacidad de análisis crítico. } \\
\text { Esta última implica: formular objetivos } \\
\text { de lectura, leer críticamente, recono- } \\
\text { cer la estructura del texto e investigar } \\
\text { el contexto del texto (condiciones de } \\
\text { producción, enfoque teórico, posiciona- } \\
\text { miento del autor, bibliografía utilizada). }\end{array}$ & $\begin{array}{l}\text { Dentro de las competencias que se consideran } \\
\text { necesarias para escribir textos académicos } \\
\text { destacan: la capacidad argumentativa y de } \\
\text { construir una voz propia, el conocimiento del } \\
\text { tema que se aborda y la capacidad investiga- } \\
\text { tiva que tiene que ver con la competencia de } \\
\text { lectura crítica (saber buscar fuentes relevan- } \\
\text { tes, saber reconocer el propósito del escrito). } \\
\text { También es importante el pensamiento crea- } \\
\text { tivo, el capital cultural con el que contemos, } \\
\text { la humildad académica, el ser ético, y saber } \\
\text { redactar de manera sencilla. }\end{array}$ \\
\hline Prácticas & $\begin{array}{l}\text { Entre las prácticas de lectura académica, } \\
\text { destacan: } \\
\text { - Establecer un objetivo de lectura para } \\
\text { rescatar las partes importantes del texto } \\
\text { Investigar contexto y autor } \\
\text { - Revisar el texto de manera gradual, } \\
\text { refiriéndose a otras fuentes cuando se } \\
\text { considere necesario } \\
\text { - Revisar las posturas teóricas que se } \\
\text { discuten en el texto } \\
\text { - De ser necesario, realizar varias lectu- } \\
\text { ras, en distintos niveles de profundidad. }\end{array}$ & $\begin{array}{l}\text { Las prácticas de escritura académica que se } \\
\text { destacan son: } \\
\text { - Definir quién será el lector } \\
\text { - Realizar investigación previa sobre el tema y } \\
\text { elaborar notas de la bibliografía revisada } \\
\text { - Generar mapas para desglosar ideas } \\
\text { - Plantear objetivos } \\
\text { - Definir un posicionamiento } \\
\text { - Contrastar resultados y teorías (en un aparta- } \\
\text { do de discusión) } \\
\text { - Establecer coherencia entre las partes del } \\
\text { escrito (revisión) } \\
\text { - Dar a leer a un tercero (revisión) }\end{array}$ \\
\hline
\end{tabular}

Elaboración propia. 


\section{Algunas reflexiones finales}

A partir del análisis de los resultados de esta primera etapa de trabajo, he podido identificar la presencia de los dos tipos de concepciones de lectura y escritura académicas: transmisivas y transaccionales. Estas concepciones no se presentan como opuestas, ni de modo puro en una misma persona, sino que existe mayor carga de una u otra. Esto coincide con lo descubierto en otras investigaciones (White, Bruning, 2004; Villalón, Mateos, 2009; Villalón, 2010; Hernández, Rodríguez, 2018; Gaeta et al., 2020) acerca de que ambos tipos de concepciones pueden coexistir en un mismo sujeto.

Asimismo, en los párrafos anteriores se reportaron, de manera general, algunas de las prácticas de lectura y escritura de docentes y estudiantes del posgrado, correspondientes con ambos tipos de concepciones. A pesar de las diferencias entre concepciones, se pueden identificar prácticas similares como, por ejemplo, referirse a otras fuentes para mejorar la comprensión de un texto o revisar la coherencia del texto escrito. Las prácticas que se corresponden con las concepciones transaccionales reflejan un uso más crítico y metarreflexivo de lectura y la escritura. Esto coincide con lo indicado por White y Bruning (2004), quienes, al evaluar las creencias de escritura, revelaron que los estudiantes con creencias transaccionales producían composiciones escritas donde integraban contenido crítico e ideas personales.

En general, se corroboró que aquellos con concepciones transmisivas se predisponen como lectores y escritores pasivos en lugar de constructores activos de significado, como en el caso de quienes cuentan con mayor carga de concepciones transaccionales (cfr. Schraw, Bruning, 1996; White, Bruning, 2004).

Si bien estos resultados preliminares se complementarán con la segunda etapa metodológica propuesta en el diseño de esta investigación, es pertinente observar que -como ya lo señalaron Gaeta et al., 2020- hacen falta más indagaciones sobre las prácticas y concepciones de lectura y escritura que se desarrollan en las distintas disciplinas del contexto académico.

\section{Referencias}

Arnoux, E. (2009). Presentación. En: Arnoux, E. (ed.). Escritura y producción de conocimiento en las carreras de posgrado. Santiago: Arcos editor.

Benedetto, L. (2003). La complementariedad metodológica en la investigación didáctica. En Medina Rivilla, A.; S. Castillo Arredondo (coords.). Metodología para la realización de proyectos de investigación y tesis doctorales. México: Ed. Universitas, 228-248.

Carlino, P. (2005). Escribir, leer y aprender en la universidad: Una introducción a la alfabetización académica. Fondo de Cultura Económica.

Carlino, P. (2013). Alfabetización académica diez años después. Revista Mexicana de Investigación Educativa, 18(57), 355-381. https://tinyurl.com/y5ncewyx 
Cassany, D. (2006). Tras las líneas. Barcelona: Anagrama.

Castaño, E.; J. de la Fuente (2013). Lenguaje del arquitecto: diagnóstico y propuestas académicas. Revista de Docencia Universitaria, 11(3). https://doi.org/10.4995/redu.2013.5530

Castelló, M.; G. Bañales; N. Vega (2011). Leer múltiples documentos para escribir textos académicos en la universidad: o cómo aprender a leer y escribir en el lenguaje de las disciplinas. Pro-Posiçōes, 22(1). 97-114. https://doi.org/10.1590/\$0103-73072011000100009

Castelló, M.; M. Mateos; N. Castells; A. Iñesta; I. Cuevas; I. Solé (2012). Academic Writing Practices in Spanish Universities [Prácticas de escritura académica en universidades españolas]. Electronic Journal of Research in Educational Psychology, 10(2), 569-590.

Castelló, M.; M. Mateos (2015). Faculty and Student Representations of Academic Writing at Spanish Universities [Las representaciones de profesores y estudiantes sobre la escritura académica en las universidades españolas]. Cultura y Educación, 27(3), 477-503.

https://doi.org/10.1080/11356405.2015.1072357

CEPAL (2018). Anuario estadístico de América Latina y El Caribe. https://tinyurl.com/8mfa79bn

Chois, P.; L. Jaramillo (2016). La investigación sobre la escritura en posgrado: estado del arte. Lenguaje, 44(2), 227-259. https://tinyurl.com/d6z787xi

Cisterna, F. (2005). Categorización y triangulación como procesos de validación del conocimiento en investigación cualitativa. Theoria, 14(1), 61-71.

http://www.redalyc.org/articulo.oa?id=29900107

Díaz, F. (2006). Enseñanza situada: vínculo entre la escuela y la vida. México: McGraw-Hill Interamericana.

Difabio, H. (2013). Evaluación de las concepciones de escritura académica en doctorandos en educación. Revista Actualidades Investigativas en Educación, 13(3). 1-21.

Espino, S. (2015). La enseñanza de estrategias de escritura y comunicación de textos científicos y académicos a estudiantes de posgrado. Revista Mexicana de Investigación Educativa, 20(66), 959-976. https://tinyurl.com/zzfddxj2

Ferreiro, E. (2007). Las inscripciones de la escritura. Conferencia pronunciada en la Universidad de La Plata, Argentina. http://bit.ly/2Zbvwy1

Gaeta, M.; M. Zanotto; G. González-Ocampo (2020). Concepciones de escritura académica en estudiantes de medicina. Revista de Investigación Educativa de la REDIECH.

https://doi.org/10.33010/ie rie rediech.v11i0.855

Gee, J. P. (2010). A Situated-Sociocultural Approach to Literacy and Technology [Un acercamiento situado y sociocultural a la literacidad y la tecnología]. En Baker, E. A. (ed.). The New Literacies: Multiple Perspectives on Research and Practice. Nueva York: The Guilford Press, 165-189.

Hernández, G. (2008). Teorías implícitas de la lectura y conocimiento metatextual. Revista Mexicana de Investigación Educativa, 13(38), 737-771.

http://www.scielo.org.mx/pdf/rmie/v13n38/v13n38a4.pdf

Diólo oos

sobre Educación 
Hernández, G. (2012). Teorías implícitas de escritura en estudiantes pertenecientes a dos comunidades académicas distintas. Perfiles Educativos, 24(136), 42-62. https://tinyurl.com/yzd7x278

Hernández, G.; E. Rodríguez (2018). Creencias y prácticas de escritura. Comparación entre distintas comunidades académicas. Revista Mexicana de Investigación Educativa. 23(79). https:// tinyurl.com/mt5ytzj

Horkheimer, M. y Adorno, T. (1994). Dialéctica de la ilustración. Fragmentos filosóficos. Madrid: Trotta.

INEE (2011). ¿Qué saben los estudiantes al término de la educación media superior? La Educación Media Superior en México. https://tinyurl.com/rasdt8bw

Lave, J.; E. Wenger (1991). Situated Learning. Legitimate Peripheral Participation. [Aprendizaje situado. Participación periférica legítima]. Cambridge: University of Cambridge Press.

López, G. (2013). Prácticas disciplinares, prácticas escolares. Qué son las disciplinas académicas y cómo se relacionan con la educación formal en las ciencias y en las humanidades. Revista Mexicana de Investigación Educativa, 18(7), 383-412.

http://www.scielo.org.mx/pdf/rmie/v18n57/v18n57a4.pdf

Marcuse, H. (1993). El hombre unidimensional. Ensayo sobre la ideología de la sociedad industrial avanzada. Barcelona: Editorial Planeta-Agostini.

Martinic, S. (2006). El estudio de las representaciones y el análisis estructural del discurso. En Canales, M. (coord.). Metodologías de investigación social. Introducción a los oficios. Santiago: LOM.

Monje, C. (2011). Metodología de investigación cuantitativa y cualitativa. Guía didáctica. Colombia: Universidad Surcolombiana.

Morales, O.; D. Cassany (2008). Leer y escribir en la universidad: Hacia la lectura y la escritura crítica de géneros científicos. Revista Memoralia, (5), 69-82.

Navarro, F. (2017). De la alfabetización académica a la alfabetización disciplinar. En Ibáñez, R.: C. González (eds.). Alfabetización disciplinar en la formación inicial docente. Leer y escribir para aprender. Valparaíso: Ediciones Universitarias de Valparaíso, 7-15.

Navarro, F.; B. Mora-Aguirre (2019). Teorías implícitas sobre escritura académica y su enseñanza: contrastes entre el ingreso, la transición y el egreso universitarios. Universitas Psychologica, 18(3), 1-16. https://doi.org/10.11144/Javeriana.upsy18-3.tiea

Navarro, F.; F. Uribe; P. Lovera; E. Sologuren (2020). Encuentros con la escritura en el ingreso a la educación superior. Representaciones sociales de los estudiantes en seis áreas de conocimiento. Revista Ibérica, (38), 75-98. https://tinyurl.com/7ctsccn8

Padilla, C. (2019). Escritura epistémico-argumentativa e identidad académica en estudiantes doctorales de humanidades: trayectorias previas, revisión colaborativa y perfiles de escritor. Traslaciones. Revista Latinoamericana de Lectura y Escritura, 6(11), 86-115. http://hdl.handle.net/11336/94421 
Peredo, A. (2016). Concepciones de escritura académica y aprendizaje en estudiantes universitarios. En González de la Torre, Y.; A. Ponce Rojo (eds.). Lectura, escritura y matemáticas: Una mirada desde los estudios de Literacidad. México: Universidad de Guadalajara.

Pipkin, M.; M. Reynoso (2010). Prácticas de lectura y escritura académicas. Argentina: Comunicarte. Sánchez, M. (2016). El Área de Primera Lengua de la Universidad de las Américas Puebla, México. En Natale, L.; D. Stagnaro (comps.). Alfabetización académica: Un camino hacia la inclusión en el nivel superior. Argentina: Ediciones UNGS, 47-74.

Schraw, G.; R. Bruning (1996). Readers'Implicit Models of Reading. [Modelos implícitos de lectura en lectores]. Reading Research Quarterly, 31(3), 290-305. https://doi.org/0.1598/RRQ.31.3.4

Seide, M.; L. Natale (2017). Concepciones de la escritura y de la alfabetización académica en devoluciones escritas de docentes universitarios: un estudio contrastivo. SCRIPTA, Belo Horizonte, 21(43), 236-258. https://dialnet.unirioja.es/servlet/articulo?codigo $=6230673$

Solé, I.; M. Mateos; M. Miras; E. Martín; I. Cuevas; N. Castells; M. Gràcia (2005). Lectura, escritura y adquisición de conocimientos en educación secundaria y educación universitaria. Infancia y Aprendizaje, 28(3), 329-347. https://doi.org/10.1174/0210370054740241

Villalón, R. (2010). Las concepciones de los estudiantes sobre la escritura académica. Tesis doctoral. Universidad Autónoma de Madrid, España. http://www.tdx.cat/handle/10803/50700

Villalón, R.; M. Mateos (2009). Concepciones del alumnado de secundaria y universidad sobre la escritura académica. Infancia y Aprendizaje, (32), 219-232.

https://doi.org/10.1174/021037009788001761

Villaseñor, V. Y. (2013). Hacia una didáctica de la escritura académica en la universidad. Reencuentro, (66), 90-101. https://tinyurl.com/bpnwwmr4

Wenger, E. (1998). Communities of Practice: Learning, Meaning, and Identity (Learning in Doing: Social, Cognitive and Computational Perspectives). [Comunidades de prácticas: Aprendizaje, significado e identidad]. Cambridge: Cambridge University Press.

White, M. J.; R. Bruning (2004). Implicit Writing Beliefs and their Relation to Writing Quality. [Creencias implícitas sobre escritura y su relación con la calidad de escritura]. Contemporary Educational Psychology, (30), 166-189. https://doi.org/10.1016/j.cedpsych.2004.07.002

Zanotto, M. (2018). Concepciones de escritura académica y aprendizaje en estudiantes universitarios. En González de la Torre, Y.; A. Ponce Rojo (eds.). Lectura, escritura y matemáticas: Una mirada desde los estudios de Literacidad. México: Universidad de Guadalajara, 87-107.

Zavala, V. (2011). La escritura académica y la agencia de los sujetos. Cuadernos Comillas, (1), 6781. https://www.fundacioncomillas.es/cuadernos-comillas/cuadernos/el-espanol-escritoen-contextos-contemporaneos/pdf/cuaderno.pdf 